
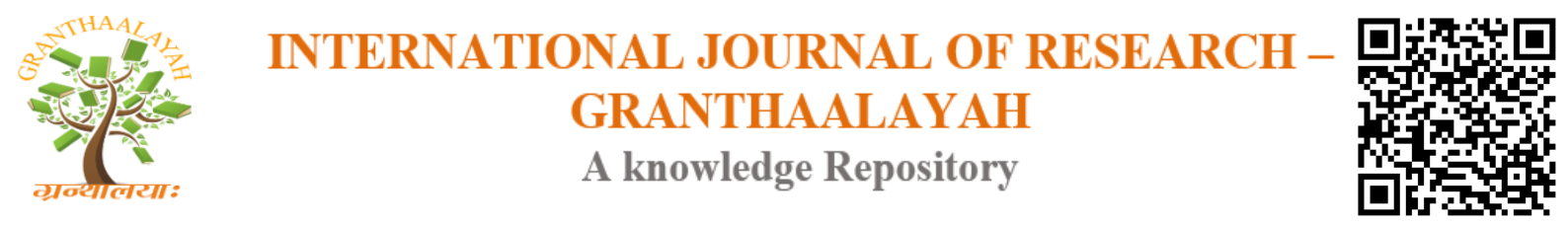

Management

\title{
A STUDY ON STRESS MANAGEMENT AMONG SELF FINANCING COLLEGE FACULTIES OF CALICUT DISTRICT
}

\author{
Swathikishore $^{* 1}$, Ms.Baby ${ }^{2}$ \\ ${ }^{* 1}$ Research Scholar, M.Phil Commerce, CMS College of Science \& Commerce, India \\ ${ }^{2}$ Assistant Professor, CMS College of Science \& Commerce, India
}

\begin{abstract}
Stress is a term generally used to define state of mind of tensions or tiredness usually associated with work overload or overly demanding work. Stress is ordinary phenomenon in an individual's daily life. Stress refers to the anxiety from the conflict between our external environment and us, leading to emotional and physical pressure. The main aim of the study is to identify the stress level of faculty members working in self-financing colleges situated in Calicut District. The study is conducted on Faculties of Self finance colleges. Faculties from many self-financing colleges are taken consideration and the sample size is 200. Simple random sampling was adopted for this study. The findings stated that majority $55.5 \%$ of faculties having stress related symptoms and $21.5 \%$ of faculties doing Meditation and Exercise to reduce stress. The study concluded that the most important causes for stress among faculties are overloaded working hours, role in the performance of job, student discipline, innovations in the higher education field, career development, funding policies, problems arise from administration side, feeling of angry, whipping by finished tiredness and unfit to move in work are considered as the essential results of stress among the Arts and Science school instructors.
\end{abstract}

Keywords: Stress; Stress Management; Self Financing; College Facilities; Calicut.

Cite This Article: Swathikishore, and Ms.Baby. (2017). "A STUDY ON STRESS MANAGEMENT AMONG SELF FINANCING COLLEGE FACULTIES OF CALICUT DISTRICT." International Journal of Research - Granthaalayah, 5(11), 149-157. https:// doi.org/10.29121/granthaalayah.v5.i11.2017.2338.

\section{Introduction}

Stress alludes to the strain from the contention between our outside condition and us, prompting enthusiastic and physical pressure. There is both positive and negative stress, contingent upon every individual's special view of the strain between the two powers. In the work environment, it can serve to upgrade a person's inspiration, execution, fulfilment and individual accomplishment. Teaching profession was once seen as a 'low-stress occupation' and they have been begrudged for residency, light workload, adaptability and different lives, for example, remote treks for study and meeting. Notwithstanding, some current investigations recommend that college staff is the 
most focused on work related gathering. To discover a sentiment about educating and taking care of understudies, to discover the anxiety makers, to the investigation of sharing anxiety issues, to discover systems connected by the College faculties for overseeing stress.

Stress is unavoidable piece of life because of expanding workload and complexities in day by day life. Presently a-days the world is said to be universe of accomplishment and furthermore the universe of stress. Stress is anyplace and all over, regardless of whether it is in family, companions, business, organization or society. The investigation of concentrates identified with work push, uncovered that worry among instructors are abnormal state in the present situation. Right from birth to death, every single individual presented to push. In this present examination, the examiner investigated the apropos factors identifying with Job Stress among Teachers by checking on the significant written works from the past investigations. Educating is likewise one of the upsetting callings like numerous different callings. They need to play out various parts and duties. Not just this, they need to keep up welcoming association with Management Principal and Colleagues. On work stress can be named as Physical and Emotional reactions that happen when the necessities of the occupation don't coordinate with the capacities, assets or requirements of the Institutions.

\subsection{Statement of the Problem}

This investigation is critical in light of the fact that it will fill in as an eye opener to the staff and executives that any type of stress one will experience ought not be underestimated and that it could hamper the execution of the personnel on the off chance that it won't be tended to right on time. The examination could be a decent wellspring of data for arranging an esteem framework keeping in mind the end goal to enable the employees to enhance their educating execution. Likewise, they could create methods on the best way to oversee stresses that they may understanding by leading conceivable keeping preparing project and stress administration classes. It would help them in evaluating the qualities and shortcomings of the workforce and they could give bits of knowledge in figuring approaches to overhaul the showing execution of the staff. The outcomes would help them really recognize their powerless and solid focuses in this way discover ways and intends to enhance their shortcomings and underwrite their qualities so as to upgrade their showing strategies and procedures. Understudies on one hand, since they are the beneficiaries of the evaluative procedure, the productivity and adequacy of the showing execution of the personnel would enhance the understudies' scholastic execution.

\subsection{Objectives of the Study}

1) To find the stress level of faculty members working in self-financing colleges situated in Calicut District.

2) To identify various influential factors of stress among faculty members.

3) To analyse strategies utilized by the College faculties to oversee work stress.

\subsection{Limitations of the Study}

1) The study is only for Self-financing colleges at Calicut district and cannot be generalized for the other districts. 
2) The study has biased response for certain questionnaire because they had fear of further consequences.

3) The study was conducted only for a time period of six months, hence all the aspects of stress management was not covered.

4) Busy schedule of the faculties was hindrance to collect data's. So they dont find enough time to read the questionnaire carefully.

5) Unwillingness and fear for unknown reasons on the part of the respondents to reveal information was one of the major constraints.

6) The study had to be confined only to 200 faculties out of 300 .

\section{Research Methodology Used}

\begin{tabular}{|l|l|}
\hline Research Design & Descriptive research \\
\hline Target population & 250 Self-financing College faculties in Calicut District \\
\hline Sampling size & 200 \\
\hline Period of study & 6 Months \\
\hline Data Collection Tool & Primary and Secondary \\
\hline Primary data & $\begin{array}{l}\text { Questionnaire with personal questions in closed ended form and study } \\
\text { related questions in 5 point Likert scale }\end{array}$ \\
\hline Secondary data & Websites and magazines. \\
\hline Sampling Technique & Simple random sampling \\
\hline Statistical Tools & Percentage Analysis, Chi square and ANOVA \\
\hline
\end{tabular}

\section{Analysis and Results}

Table 3.1: Distribution Of Samples Based On Variables

\begin{tabular}{|c|c|c|c|c|c|}
\hline S.NO & Category & Subgroups & No. & $\%$ & Total \\
\hline \multirow{4}{*}{1.} & \multirow{4}{*}{ Age } & Above 20 years & 43 & 21.5 & \multirow{4}{*}{200} \\
\hline & & $25-35$ years & 45 & 22.5 & \\
\hline & & $36-45$ years & 50 & 25 & \\
\hline & & Above 45 years & 62 & 31 & \\
\hline \multirow[t]{2}{*}{2.} & \multirow[t]{2}{*}{ Gender } & Male & 102 & 51 & \multirow[t]{2}{*}{200} \\
\hline & & Female & 98 & 49 & \\
\hline \multirow{4}{*}{3.} & \multirow[t]{4}{*}{ Designation } & Assistant Professor & 50 & 25 & \multirow[t]{4}{*}{200} \\
\hline & & Associate Professor & 50 & 25 & \\
\hline & & Professor & 57 & 28.05 & \\
\hline & & Lecturer & 43 & 21.5 & \\
\hline \multirow[t]{3}{*}{4.} & \multirow[t]{3}{*}{ General health for past 3 years } & Good & 70 & 35 & \multirow[t]{3}{*}{200} \\
\hline & & Reasonable & 71 & 35.5 & \\
\hline & & Poor & 59 & 29.5 & \\
\hline \multirow[t]{4}{*}{5.} & \multirow[t]{4}{*}{ Average No. of hours per week } & $10-15$ hours & 52 & 26 & \multirow[t]{4}{*}{200} \\
\hline & & $15-20$ hours & 54 & 27 & \\
\hline & & $20-25$ hours & 42 & 21 & \\
\hline & & $25-30$ hours & 52 & 26 & \\
\hline 6. & Steps taken to reduce stress & Meditation & 43 & 21.5 & 200 \\
\hline
\end{tabular}




\begin{tabular}{|c|c|c|c|c|c|}
\hline & & Exercise & 43 & 21.5 & \\
\hline & & Yoga & 42 & 21 & \\
\hline & & Movies & 38 & 19 & \\
\hline & & Outing & 34 & 17 & \\
\hline 7. & Leave taken in past 12 months & Yes & 94 & 47 & 200 \\
\hline & & No & 106 & 53 & \\
\hline 8. & Stress Related symptoms & Yes & 111 & 55.5 & 200 \\
\hline & & No & 89 & 44.5 & \\
\hline
\end{tabular}

Table 3.2: Chi Square Analysis

\begin{tabular}{|c|c|c|c|c|c|}
\hline Hypothesis & $\begin{array}{l}\text { Calculated } \\
\text { Value }\end{array}$ & $\begin{array}{l}\text { Table } \\
\text { Value }\end{array}$ & $\begin{array}{l}\text { Degrees } \\
\text { Of } \\
\text { Freedom }\end{array}$ & $\begin{array}{l}\text { Null } \\
\text { Hypothesis }\end{array}$ & Remarks \\
\hline $\begin{array}{l}\mathbf{H}_{\mathbf{0}}(\mathbf{1}) \text { : There is no significant } \\
\text { relationship between Age and } \\
\text { Stress Symptoms. }\end{array}$ & 1.569 & 12.59 & 6 & Accepted & $\begin{array}{l}\text { Not } \\
\text { Significant }\end{array}$ \\
\hline $\begin{array}{l}\mathbf{H}_{\mathbf{0}} \text { (2): There is no significant } \\
\text { relationship between Age and } \\
\text { Teaching New Courses. }\end{array}$ & 7.051 & 21.03 & 12 & Accepted & $\begin{array}{l}\text { Not } \\
\text { Significant }\end{array}$ \\
\hline $\begin{array}{l}\mathbf{H}_{\mathbf{0}}(\mathbf{3}) \text { : There is no significant } \\
\text { relationship between Age and } \\
\text { Long Working Hours. }\end{array}$ & 7.992 & 16.92 & 9 & Accepted & $\begin{array}{l}\text { Not } \\
\text { Significant }\end{array}$ \\
\hline $\begin{array}{l}\mathbf{H}_{\mathbf{0}}(\mathbf{4}) \text { : There is no significant } \\
\text { relationship between Age and } \\
\text { Lack of particpation in decision } \\
\text { making }\end{array}$ & 14.265 & 16.92 & 9 & Accepted & $\begin{array}{l}\text { Not } \\
\text { Significant }\end{array}$ \\
\hline $\begin{array}{l}\mathbf{H}_{\mathbf{0}}(\mathbf{5}) \text { : There is no significant } \\
\text { relationship between Age and } \\
\text { Lack of involvement }\end{array}$ & 15.320 & 16.92 & 9 & Accepted & $\begin{array}{l}\text { Not } \\
\text { Significant }\end{array}$ \\
\hline $\begin{array}{l}\mathbf{H}_{0}(\mathbf{6}) \text { : There is no significant } \\
\text { relationship between Age and } \\
\text { Lack of career development } \\
\text { opportunity. }\end{array}$ & 7.764 & 16.92 & 9 & pted & $\begin{array}{l}\text { Not } \\
\text { Significant }\end{array}$ \\
\hline $\begin{array}{l}\mathbf{H}_{0}(\mathbf{7}) \text { : There is no significant } \\
\text { relationship between Age and } \\
\text { High degree of uncertainity about } \\
\text { work. }\end{array}$ & 3.289 & 16.92 & 9 & Accepted & $\begin{array}{l}\text { Not } \\
\text { Significant }\end{array}$ \\
\hline $\begin{array}{l}\mathbf{H}_{\mathbf{0}}(\mathbf{8}) \text { : There is no significant } \\
\text { relationship between Age and } \\
\text { Feeling work not valued. }\end{array}$ & 8.508 & 16.92 & 9 & Accepted & $\begin{array}{l}\text { Not } \\
\text { Significant }\end{array}$ \\
\hline $\begin{array}{l}\mathbf{H}_{\mathbf{0}}(\mathbf{9}) \text { : There is no significant } \\
\text { relationship between Age and } \\
\text { Lack of Physical Energy. }\end{array}$ & 7.416 & 16.92 & 9 & Accepted & $\begin{array}{l}\text { Not } \\
\text { Significant }\end{array}$ \\
\hline $\begin{array}{l}\mathbf{H}_{\mathbf{0}}(\mathbf{1 0}) \text { : There is no significant } \\
\text { relationship between Age and } \\
\text { Feeling Tired while awake. }\end{array}$ & 6.462 & 16.92 & 9 & Accepted & $\begin{array}{l}\text { Not } \\
\text { Significant }\end{array}$ \\
\hline
\end{tabular}


DOI: 10.5281/zenodo.1069416

\begin{tabular}{|l|l|l|l|l|l|}
\hline $\begin{array}{l}\mathbf{H}_{\mathbf{0}} \text { (11): There is no significant } \\
\text { relationship between Age and } \\
\text { Working time is late. }\end{array}$ & 13.386 & 16.92 & 9 & Accepted & $\begin{array}{l}\text { Not } \\
\text { Significant }\end{array}$ \\
\hline $\begin{array}{l}\mathbf{H}_{\mathbf{0}} \text { (12): There is no significant } \\
\text { relationship between Age and } \\
\text { Cannot solve problems. }\end{array}$ & 5.932 & 16.92 & 9 & Accepted & $\begin{array}{l}\text { Not } \\
\text { Significant }\end{array}$ \\
\hline $\begin{array}{l}\mathbf{H}_{\mathbf{0}} \text { (13): There is no significant } \\
\text { relationship between Age and } \\
\text { Spending time with family. }\end{array}$ & 4.837 & 16.92 & 9 & Accepted & $\begin{array}{l}\text { Not } \\
\text { Significant }\end{array}$ \\
\hline $\begin{array}{l}\mathbf{H}_{\mathbf{0}} \text { (14): There is no significant } \\
\text { relationship between Age and } \\
\text { Steps taken to reduce stress. }\end{array}$ & 9.797 & 21.03 & 12 & Accepted & $\begin{array}{l}\text { Not } \\
\text { Significant }\end{array}$ \\
\hline $\begin{array}{l}\mathbf{H}_{\mathbf{0}} \text { (15): There is no significant } \\
\text { relationship between Designation } \\
\text { and Leave in past } 12 \text { months. }\end{array}$ & 1.528 & 7.82 & 3 & Accepted & $\begin{array}{l}\text { Not } \\
\text { Significant }\end{array}$ \\
\hline $\begin{array}{l}\mathbf{H}_{\mathbf{0}} \text { (16): There is no significant } \\
\text { relationship between Designation } \\
\text { and Stress related symptoms. }\end{array}$ & 5.942 & 7.82 & 3 & Accepted & $\begin{array}{l}\text { Not } \\
\text { Significant }\end{array}$ \\
\hline $\begin{array}{l}\mathbf{H}_{\mathbf{0}} \text { (17): There is no significant } \\
\text { relationship between Designation } \\
\text { and Efforts not valued. }\end{array}$ & 8.939 & 16.92 & 9 & Accepted & $\begin{array}{l}\text { Not } \\
\text { Significant }\end{array}$ \\
\hline $\begin{array}{l}\mathbf{H}_{\mathbf{0}} \text { (18): There is no significant } \\
\text { relationship between Designation } \\
\text { and Lack of Management } \\
\text { Support. }\end{array}$ & 11.459 & 16.92 & 9 & Accepted & $\begin{array}{l}\text { Not } \\
\text { Significant }\end{array}$ \\
\hline $\begin{array}{l}\mathbf{H}_{\mathbf{0}} \text { (19): There is no significant } \\
\text { relationship between Designation } \\
\text { and Cannot solve problems. }\end{array}$ & 11.519 & 16.92 & 9 & Accepted & $\begin{array}{l}\text { Not } \\
\text { Significant }\end{array}$ \\
\hline $\begin{array}{l}\mathbf{H}_{\mathbf{0}} \text { (20): There is no significant } \\
\text { relationship between Designation } \\
\text { and Steps taken to reduce stress. }\end{array}$ & 17.451 & 21.03 & 12 & Accepted & $\begin{array}{l}\text { Not } \\
\text { Significant }\end{array}$ \\
\hline
\end{tabular}

Table 3.3: Anova Analysis

\begin{tabular}{|c|c|c|c|c|}
\hline Hypothesis & $\begin{array}{l}\text { F- } \\
\text { value }\end{array}$ & Sig. & NH & $\mathbf{R}$ \\
\hline $\begin{array}{l}\mathbf{H}_{\mathbf{0}} \text { (21): There is no significant difference } \\
\text { between Age and Working hours of faculties. }\end{array}$ & 1.718 & 0.165 & Accepted & $\begin{array}{l}\text { Not } \\
\text { Significant }\end{array}$ \\
\hline $\begin{array}{l}\mathbf{H}_{\mathbf{0}} \text { (22): There is no significant difference } \\
\text { between Gender and Working hours of faculties. }\end{array}$ & 0.254 & 0.615 & Accepted & $\begin{array}{l}\text { Not } \\
\text { Significant }\end{array}$ \\
\hline $\begin{array}{l}\mathbf{H}_{\mathbf{0}} \text { (23): There is no significant difference } \\
\text { between Designation and Working hours of } \\
\text { faculties. }\end{array}$ & 0.793 & 0.499 & Accepted & $\begin{array}{l}\text { Not } \\
\text { Significant }\end{array}$ \\
\hline $\begin{array}{l}\mathbf{H}_{\mathbf{0}}(\mathbf{2 4 )} \text { : There is no significant relationship } \\
\text { between Age and Lack of particpation in decision } \\
\text { making }\end{array}$ & 14.265 & 16.92 & Accepted & $\begin{array}{l}\text { Not } \\
\text { Significant }\end{array}$ \\
\hline
\end{tabular}




\begin{tabular}{|l|l|l|l|l|l|}
\hline $\begin{array}{l}\mathbf{H}_{\mathbf{0}} \text { (25): There is no significant relationship } \\
\text { between Age and Lack of involvement }\end{array}$ & 15.320 & 16.92 & Accepted & $\begin{array}{l}\text { Not } \\
\text { Significant }\end{array}$ \\
\hline
\end{tabular}

Table 3.3.1: $\mathbf{H}_{\mathbf{0}}$ (26): There is no significant difference between Age and Stress Symptoms in

\begin{tabular}{|l|l|l|l|l|l|l|l|l|l|}
\hline \multirow{2}{*}{} & Irritated & Angry & $\begin{array}{l}\text { Frustrate } \\
\text { d }\end{array}$ & Helpless & Anxious & $\begin{array}{l}\text { Unable to } \\
\text { Depresse } \\
\text { d }\end{array}$ & $\begin{array}{l}\text { Over } \\
\text { Concentra } \\
\text { te }\end{array}$ \\
\hline $\begin{array}{l}\text { Ag } \\
\mathbf{e}\end{array}$ & $\begin{array}{l}\text { F- } \\
\text { Value }\end{array}$ & 0.512 & 1.260 & 1.153 & 1.930 & 0.543 & 1.169 & 0.447 & 1.205 \\
\cline { 2 - 9 } & Sig & 0.675 & 0.289 & 0.329 & 0.126 & 0.653 & 0.323 & 0.720 & 0.309 \\
\hline & NH & Accepted & Accepted & Accepted & Accepted & Accepted & Accepted & Accepted & Accepted \\
\cline { 2 - 9 } & $\begin{array}{l}\text { Remar } \\
\text { ks }\end{array}$ & $\begin{array}{l}\text { Not } \\
\text { Significa } \\
\text { nt }\end{array}$ & $\begin{array}{l}\text { Not } \\
\text { Significa } \\
\text { nt }\end{array}$ & $\begin{array}{l}\text { Not } \\
\text { Significa } \\
\text { nt }\end{array}$ & $\begin{array}{l}\text { Not } \\
\text { Significa } \\
\text { nt }\end{array}$ & $\begin{array}{l}\text { Not } \\
\text { Significa } \\
\text { nt }\end{array}$ & $\begin{array}{l}\text { Not } \\
\text { Significa } \\
\text { nt }\end{array}$ & $\begin{array}{l}\text { Not } \\
\text { Significant }\end{array}$ & $\begin{array}{l}\text { Not } \\
\text { Significa } \\
\text { nt }\end{array}$ \\
\hline
\end{tabular}

Table 3.3.2: $\mathbf{H}_{\mathbf{0}}$ (27): There is no significant difference between Age and Time factor causing stress.

\begin{tabular}{|l|l|l|l|l|l|l|}
\hline & & $\begin{array}{l}\text { Dealing with } \\
\text { student } \\
\text { discipline }\end{array}$ & $\begin{array}{l}\text { Teaching new } \\
\text { courses }\end{array}$ & $\begin{array}{l}\text { Long } \\
\text { working } \\
\text { hours }\end{array}$ & Lone working & $\begin{array}{l}\text { Travelling } \\
\text { time }\end{array}$ \\
\hline \multirow{2}{*}{ Age } & F-Value & 3.149 & 0.278 & 0.434 & 0.956 & 0.871 \\
\cline { 2 - 7 } & Sig & 0.026 & 0.841 & 0.729 & 0.415 & 0.457 \\
\cline { 2 - 7 } & NH & Rejected & Accepted & Accepted & Accepted & Accepted \\
\cline { 2 - 7 } & Remarks & Significant & $\begin{array}{l}\text { Not } \\
\text { Significant }\end{array}$ & $\begin{array}{l}\text { Not } \\
\text { Significant }\end{array}$ & $\begin{array}{l}\text { Not } \\
\text { Significant }\end{array}$ & $\begin{array}{l}\text { Not } \\
\text { Significant }\end{array}$ \\
\hline
\end{tabular}

Table 3.3.3: $\mathbf{H}_{\mathbf{0}} \mathbf{( 2 8 )}$ : There is no significant difference between Gender and Relationship factor

\begin{tabular}{|c|c|c|c|c|c|c|}
\hline & & \begin{tabular}{|l} 
Dealing \\
with \\
conflictual \\
situations \\
\end{tabular} & $\begin{array}{l}\text { Lack of line } \\
\text { management } \\
\text { support }\end{array}$ & $\begin{array}{l}\text { Lack of } \\
\text { communication } \\
\text { with staff }\end{array}$ & $\begin{array}{l}\text { Lack of } \\
\text { involvement }\end{array}$ & $\begin{array}{l}\text { Feeling that } \\
\text { your work is } \\
\text { not valued }\end{array}$ \\
\hline \multirow[t]{4}{*}{ Gender } & F-Value & 0.038 & 2.044 & 0.007 & 0.013 & 0.030 \\
\hline & Sig & 0.847 & 0.154 & 0.931 & 0.910 & 0.862 \\
\hline & $\mathrm{NH}$ & Accepted & Accepted & Accepted & Accepted & Accepted \\
\hline & Remarks & $\begin{array}{l}\text { Not } \\
\text { Significant }\end{array}$ & $\begin{array}{l}\text { Not } \\
\text { Significant }\end{array}$ & $\begin{array}{l}\text { Not } \\
\text { Significant }\end{array}$ & $\begin{array}{l}\text { Not } \\
\text { Significant }\end{array}$ & $\begin{array}{l}\text { Not } \\
\text { Significant }\end{array}$ \\
\hline
\end{tabular}


Table 3.3.4: $\mathbf{H}_{\mathbf{0}}$ (29): There is no significant difference between Designation and Control factor

\begin{tabular}{|c|c|c|c|c|c|c|}
\hline & & $\begin{array}{l}\text { Lack of } \\
\text { Participation } \\
\text { in decision } \\
\text { making }\end{array}$ & \begin{tabular}{|l} 
Unable to \\
plan \\
working \\
day
\end{tabular} & $\begin{array}{l}\text { Job changes } \\
\text { without } \\
\text { consultation }\end{array}$ & $\begin{array}{l}\text { Changes in } \\
\text { terms \& } \\
\text { conditions } \\
\text { without } \\
\text { consultation. }\end{array}$ & $\begin{array}{l}\text { Insufficient } \\
\text { time for } \\
\text { research }\end{array}$ \\
\hline \multirow[t]{4}{*}{ Designation } & F-Value & 1.539 & 1.465 & 1.384 & 2.082 & 3.438 \\
\hline & Sig & 0.206 & 0.225 & 0.249 & 0.104 & 0.018 \\
\hline & $\mathrm{NH}$ & Accepted & Accepted & Accepted & Accepted & Rejected \\
\hline & Remarks & $\begin{array}{l}\text { Not } \\
\text { Significant }\end{array}$ & $\begin{array}{l}\text { Not } \\
\text { Significant }\end{array}$ & $\begin{array}{l}\text { Not } \\
\text { Significant }\end{array}$ & $\begin{array}{l}\text { Not } \\
\text { Significant }\end{array}$ & Significant \\
\hline
\end{tabular}

Table 3.3.5: $\quad \mathbf{H}_{\mathbf{0}} \mathbf{( 3 0 )}$ : There is no significant difference between Age and Physical indicator stress.

\begin{tabular}{|l|l|l|l|l|}
\hline & $\begin{array}{l}\text { Severe or Chronic Lower Back } \\
\text { Pain }\end{array}$ & $\begin{array}{l}\text { Chronic } \\
\text { Headaches }\end{array}$ & $\begin{array}{l}\text { Lack of Physical } \\
\text { Energy }\end{array}$ \\
\hline \multirow{2}{*}{ Age } & F-Value & 1.204 & 2.401 & 1.579 \\
\cline { 2 - 5 } & Sig & 0.310 & 0.069 & 0.196 \\
\cline { 2 - 5 } & NH & Accepted & Accepted & Accepted \\
\cline { 2 - 5 } & Remarks & Not Significant & Not Significant & Not Significant \\
\hline
\end{tabular}

Table 3.3.6: $\mathbf{H}_{\mathbf{0}} \mathbf{( 3 1 )}$ : There is no significant difference between Age and Sleep indicator of stress.

\begin{tabular}{|l|l|l|l|l|}
\hline & & $\begin{array}{l}\text { Trouble falling } \\
\text { Asleep }\end{array}$ & $\begin{array}{l}\text { Nightmares or } \\
\text { Bad Dreams }\end{array}$ & $\begin{array}{l}\text { While awake } \\
\text { feeling tired }\end{array}$ \\
\hline \multirow{2}{*}{ Age } & F-Value & 0.796 & 1.462 & 0.208 \\
\cline { 2 - 5 } & Sig & 0.497 & 0.226 & 0.891 \\
\cline { 2 - 5 } & NH & Accepted & Accepted & Accepted \\
\cline { 2 - 5 } & Remarks & Not Significant & Not Significant & Not Significant \\
\hline
\end{tabular}

Table 3.3.7: $\mathbf{H}_{\mathbf{0}}$ (32): There is no significant difference between Age and Behavioural indicator of stress.

\begin{tabular}{|c|c|c|c|c|}
\hline & & \begin{tabular}{|l|} 
Trouble in \\
remembering
\end{tabular} & Worry a lot & \begin{tabular}{|l|} 
Cannot \\
Problems
\end{tabular} \\
\hline \multirow[t]{4}{*}{ Age } & F-Value & 0.282 & 1.719 & 1.154 \\
\hline & Sig & 0.838 & 0.164 & 0.328 \\
\hline & $\mathrm{NH}$ & Accepted & Accepted & Accepted \\
\hline & Remarks & Not Significant & Not Significant & Not Significant \\
\hline
\end{tabular}


Table 3.3.8: $\mathbf{H}_{\mathbf{0}}$ (33): There is no significant difference between Age and Personal Habits of Faculties.

\begin{tabular}{|l|l|l|l|l|}
\hline \multirow{2}{*}{ Age } & $\begin{array}{l}\text { Spending time for } \\
\text { Hobby } \\
\text { for Family }\end{array}$ & $\begin{array}{l}\text { Spending time for } \\
\text { Entertainment }\end{array}$ \\
\hline & F-Value & 0.237 & 0.497 & 3.223 \\
\cline { 2 - 5 } & Sig & 0.870 & 0.685 & 0.024 \\
\cline { 2 - 5 } & Accepted & Accepted & Rejected \\
\cline { 2 - 5 } & Remarks & Not Significant & Not Significant & Significant \\
\hline
\end{tabular}

\section{Suggestions}

Since the health of self-financing college faculties are good and they have to imrove the health conditions. The faculties should concentrate on work in the average working period of 15 20Hrs. Faculties taken leave due to stress is $47 \%$ and they should concentrate on stress factor. Only minimum number of faculties are taken steps for reduce stress and each and every faculty should concentrate on stress level. Since there is a significant difference between designation and working hours and the head of the institution has to discuss with faculties about working hours and then it should be balanced. There is a significance difference between age and teaching new courses where young professors need more training for teaching new courses. There is lack of involvement for few faculties it should be analysed and rectified. The physical energy is major constraint in this study and it should be concentrated by faculties and it leads to more effective. There is significance between Age and working time is late. Some of the faculties could not complete their work on time and stress may be a reason for it and it should be corrected.

\section{Conclusion}

From the study, it is concluded that the most important causes for stress among faculties are overloaded working hours, role in the performance of job, student discipline, innovations in the higher education field, career development, funding policies, problems arise from administration side, feeling of angry, whipping by finished tiredness and unfit to move in work are considered as the essential results of stress among the Arts and Science school instructors. The high-flying adapting systems which are honed by the school instructors are readiness, setting needs, physical instantaneousness, changes in eating regimen, evasion and external divider procedures. The unmistakable effects of rehearsing stress adapting procedures are preoccupation of consideration prompts singular proficiency, positive deduction prompts authoritative execution, keeping away from difficult updates, delaying certain assignments, honest to goodness getting away of despised undertakings and designation of work prompts the work culture changes inside the Selffinancing college faculties.

The comparative study of stress among self-financing college faculties have shown that all the faculty members employed in various institutions are equally affected by stress; priority may be given to the self-financing college teachers because at the time of data collection the researcher observed that the self-financing college teachers are large in numbers and paid less. Generally, they are multifaceted people groups in workplace and perceived less. Subsequently, their part ought to be perceived for the prosperity of the general public. 


\section{References}

[1] Devarajan, G 2011, 'Employees stress in software industry', Ph.D Thesis, Bharathiyar University

[2] Dick, R \& Wagner, U 2001, 'Stress and Strain in Teaching: A Structural Equation Approach', British Journal of Educational Psychology, vol. 71, no. 2, pp. 243-259

[3] Sushma Suri \& Saba Rizvi, 'Mental Health and Stress among Call Centre Employees', Journal of the Indian Academy of Applied Psychology, vol. 34, no. 2, pp. 215-220

[4] Takashi Shimizu, Masanori Hiro, Norio Misima \& Shoji Nagata 2002, 'Job Stress Among Japanese Full Time Occupational Physicians', Journal of occupational Stress, vol. 44, pp. 348354

[5] Tang, CS, Au, WT, Schwarzer R \& Schmitz, G 2001, 'Mental health outcomes of job stress among Chinese teachers: Role of stress factors and burn out', Journal of organizational behaviour, vol. 22, pp. 887-901 